\title{
NEOGENE MARINE INVERTEBRATES OF TROPICAL AMERICA: A WORLD-WIDE WEB TAXONOMY DATABASE
}

BUDD, A. F., Geology Dept., University of Iowa, Iowa City, IA 52242, U.S.A; FOSTER, C. T., Jr., Geology Dept., University of Iowa, Iowa City, IA 52242, U.S.A; FORTUNATO, H., Smithsonian Tropical Research Institute, Box 2072, Balboa, Republic of Panama; GOLDEN, J., Geology Dept., University of Iowa, Iowa City, IA 52242, U.S.A; PETERSEN, R. A., Geology Dept., University of Iowa, Iowa City, IA 52242, U.S.A.

Neogene Marine Invertebrates of Tropical America ("NMITA") is a "virtual" collection on theWorld-Wide Web (http://porites.geology.uiowa.edu). It contains images and information on specimens and taxa collected as part of two large multitaxa fossil sampling programs: (1) the Panama Paleontology Project (PPP) in Panama and Costa Rica, coordinated by Jeremy Jackson and Anthony Coates at the Smithsonian Tropical Research Institute (STRI) in Panama; and (2) the Dominican Republic (DR) project, coordinated by Peter Jung and John Saunders at the Natural History Museum in Basel, Switzerland (NMB). NMITA is designed for use in education and research in systematics and evolutionary paleontology. Partial information is currently available for hermatypic corals, gastropods, and ostracodes. Users may click on taxa to receive conventional taxonomic information on authorship, synonyms, morphology, type specimens, and distribution. Alternatively they may click on maps and stratigraphic columns to receive faunal lists for specific horizons. From these lists, the user may click on species to return to conventional taxonomic information. A simple image-based search routine is also provided to assist in identifying hermatypic coral genera. Other search routines are under development.

NMITA has been implemented using a distributed approach involving several different web servers to allow evaluation of different platforms and techniques. The main server is NCSA httpd running on an IBM RS6000 model 220 with 16MB memory and 5GB diskspace. It currently contains $\sim 230$ images stored as gif files, and the typical image size is between 100 and $500 \mathrm{~KB}$. The associated text files have been directly coded as html. Presently our development efforts focus on integrating the server with a relational taxonomic and specimen database to allow more sophisticated queries. The preliminary model for this database consists of approximately 50 entities whose relationships are patterned after the Information Model for Biological Collections of the Association for Biological Collections. The ASC model has been enhanced by linking entities related to diagnostic morphologic characters to TaxonName-Use and by linking illustration entities to entities containing specimen, morphology, locality, and stratigraphy information. 